\title{
Magnetization dynamics in synthetic ferromagnetic thin films
}

\author{
H. J. Waring $\odot,,^{1, *}$ Y. Li $\odot,{ }^{1}$ C. Moutafis $\odot,{ }^{1}$ I. J. Vera-Marun $\odot,{ }^{2}$ and T. Thomson $\odot^{1, *}$ \\ ${ }^{1}$ Nano-Engineering and Spintronic Technologies (NEST), Department of Computer Science, University of Manchester, \\ Manchester, United Kingdom \\ ${ }^{2}$ Department of Physics and Astronomy, University of Manchester, Manchester, United Kingdom
}

(Received 18 January 2021; revised 27 April 2021; accepted 28 April 2021; published 16 July 2021)

\begin{abstract}
Synthetic ferromagnets (SFMs) possess the same layer structure found in the widely studied synthetic antiferromagnets. This consists of two ferromagnetic (FM) layers separated by a nonmagnetic (NM) spacer forming the structure $\mathrm{FM}_{1} / \mathrm{NM} / \mathrm{FM}_{2}$, but SFMs describe the case where the interlayer exchange coupling promotes the parallel alignment of the magnetizations of the FM layers. The frequency and phase of the dynamic response of these structures depends sensitively on the interlayer exchange coupling as well as on the individual layer magnetizations. Through experiments and numerical simulations, we show that the dynamic response of the two ferromagnetic layers has an orthogonal dependence on the difference in layer magnetization and interlayer coupling allowing both parameters to be determined accurately. In addition, we are able to obtain the phases of the resonant modes, a hitherto challenging measurement, and thus show that the conventional acoustic and optical description does not fully capture the intricacies of SFM dynamics. These findings are directly applicable to the creation of tailored SFMs for spintronic devices such as STT/SOT-MRAM where magnetization and interlayer coupling are key parameters.
\end{abstract}

DOI: 10.1103/PhysRevB.104.014419

\section{INTRODUCTION}

The dynamic response of magnetically ordered materials provides a sensitive method to probe fundamental magnetic properties including saturation magnetization, anisotropy, and exchange interactions [1-3]. Recently, there has been a considerable interest in exploiting the high frequency dynamics observable in ultrathin (sub-10 nm) magnetic films for spintronic applications [4-6]. Research in this area has focused upon material systems including thin ferromagnetic/heavy metal bilayers $[7,8]$, where a combination of the spin Seebeck and inverse spin Hall effect lead to broadband $\mathrm{THz}$ emission of electromagnetic radiation and antiferromagnets where intrinsically large exchange coupling leads to dynamic responses in the 100's GHz range [9-11]. An alternative to natural ferromagnetic and antiferromagnetic materials are magnetic multilayers, which offer a route to create advanced atomically engineered high frequency material systems with properties that can be tailored through the individual layers and their magnetic interactions.

The phenomena of interlayer exchange coupling in such artificially layered structures underpins many spintronic technologies and has been a rich area of investigation $[2,12,13]$.

\footnotetext{
*Corresponding authors: harry.waring@manchester.ac.uk; thomas.thomson@manchester.ac.uk

Published by the American Physical Society under the terms of the Creative Commons Attribution 4.0 International license. Further distribution of this work must maintain attribution to the author(s) and the published article's title, journal citation, and DOI.
}

An interlayer exchange interaction was demonstrated in the mid-1980s independently by Grünberg [14] and Fert [15] where antiferromagnetic coupling between $\mathrm{Fe} / \mathrm{Cr}$ layers was observed, a discovery that is widely lauded as the birth of spintronics [16,17]. The interlayer exchange coupling strength $\left(J_{\mathrm{IEC}}\right)$ is mediated by the oscillatory shortrange Ruderman-Kittel-Kasuya-Yosida (RKKY) interaction in trilayer structures of ferromagnetic $\left(\mathrm{FM}_{1}\right) /$ nonmagnetic $(\mathrm{NM}) / \mathrm{FM}_{2}$ layers and promotes a $\mathrm{FM}\left(\mathrm{J}_{\mathrm{IEC}}>0\right)$ or antiferromagnetic $(\mathrm{AF})\left(J_{\mathrm{IEC}}<0\right)$ alignment depending on spacer layer thickness as shown in Fig. 1 [18-21]. The presence of an interlayer exchange coupling results in magnetic systems with significantly different static and dynamic magnetic properties compared to those of the constituent layers [22]. To date, much attention has been focused upon synthetic antiferromagnets (SAFs) and the ramifications of AF coupling on the dynamics of these systems [12,13,22-24], where the resonance frequency is intermediate between natural ferromagnetic and antiferromagnetic ordered materials. Despite having been widely utilized in quasistatic spintronic devices $[25,26]$, the underlying dynamics and resonant physics of their synthetic ferromagnetic counterparts remains relatively unexplored.

For a synthetic ferromagnet (SFM) it has been shown that in the case where there is a difference in the magnetization of the layers $\left(\Delta M=M_{\mathrm{s}, 1}-M_{\mathrm{s}, 2}\right)$ and the presence of a weak FM interlayer exchange coupling a double resonance is obtained $[2,25]$. Reports have shown that the double resonance consists of a lower frequency acoustic mode (AM) and a higher frequency optic mode (OM) as shown schematically in Fig. 1 (inset). The AM and OM describe the phase relationship between the two precessing magnetic layers where for the AM the layer magnetizations precess in phase and for the $\mathrm{OM}$ the 


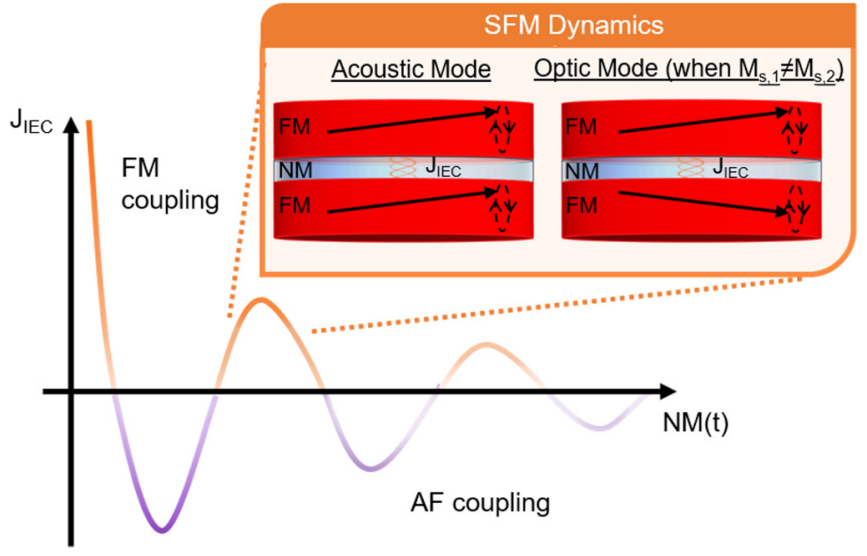

FIG. 1. The evolution of $J_{\mathrm{IEC}}$ for a $\mathrm{FM}_{1} / \mathrm{NM} / \mathrm{FM}_{2}$ trilayer. $J_{\mathrm{IEC}}$ oscillates between promoting a FM or AF layer magnetization alignment with increasing NM layer thickness. Typically, the first FM maximum occurs close to $1 \mathrm{~nm}$ with the magnetic layers decoupled at approximately $3 \mathrm{~nm}$. The inset shows the resonant dynamics of a SFM system. An optic mode is seen in addition to the acoustic mode only in cases where $M_{\mathrm{s}, 1}$ and $M_{\mathrm{s}, 2}$ are different.

layer magnetizations precess out of phase [2,23,27]. However, there remains a significant deficiency in our understanding of SFM dynamics beyond this observation. Specifically, the effect of varying (i) the interlayer exchange coupling strength and (ii) the difference in layer magnetization on the two resonant modes of the SFM system has not been studied systematically. Moreover, the influences of these parameters on the phase difference $(\Delta \Phi)$ of the two resonances has not been explored until now. Indeed it was previously reported that in a SFM it is necessary for the constituent FM layers to possess significantly different magnetic properties for the detection of an optic mode to be possible [26], hampering the range of experiments that can be performed. This has been a severe impediment to the exploration of SFM dynamics particularly in the case where the desired system must possess similar FM layer characteristics, a key technological requirement for a range of devices such as spin transfer torque (STT) or spin orbit torque (SOT) magnetic random access memory (MRAM) $[26,28]$. In these cases the determination of the $J_{\text {IEC }}$ is vital due to its impact on the thermal stability factor and the critical switching current density required [28,29]. In this work, we address this long-standing gap in knowledge and demonstrate, using a combination of measurement and simulation, that not only can the coupling parameter and difference in layer magnetization be accurately determined, but that a rich phase relation exists between the resonances of the individual layers.

\section{SAMPLE PREPARATION AND CHARACTERIZATION}

The SFM samples were fabricated using magnetron sputtering onto $\mathrm{Si} / \mathrm{SiO}_{2}$ substrates (see Appendix A) and are comprised of a multilayer structure $\mathrm{Ta}(2 \mathrm{~nm}) /$ $\mathrm{CoFeB}(5 \mathrm{~nm}) / \mathrm{Ru}\left(t_{\mathrm{Ru}}\right) / \operatorname{CoFeB}(5 \mathrm{~nm}) / \operatorname{Pd}(4 \mathrm{~nm}) . \mathrm{Ru}$ was chosen as the spacer layer material due to the strong $J_{\text {IEC }}$ enabled by this element [13]. CoFeB is a widely used magnetic film for spintronic devices including STT-MRAM, future write heads, and wireless inductors [26,30]. Alloys of $\mathrm{CoFeB}$ have a range of desirable properties including a large saturation magnetization [30-32], high resistivity [30], and high tunnel magnetoresistance values [33-35]. Ta was used as a seed layer with $\mathrm{Pd}$ as the capping layer to inhibit oxidization of the films. The layer structure of the multilayers was determined using $\mathrm{x}$-ray reflectivity (XRR) where the data were fitted to a Fresnel model by means of the Parratt recursive algorithm [36] using the GENX software package [37] (for additional information regarding the XRR measurements of the samples see Appendix B 1 and Ref. [22]). Multilayers exhibiting magnetic properties consistent with a positive $J_{\text {IEC }}$ on the first FM coupling peak were found in the case of $t_{\mathrm{Ru}}=1.03,1.07$, and 1.10 nm, referred to as SFM1, SFM2, and SFM3 respectively. A single CoFeB layer, with a structure $\mathrm{Ta}(2 \mathrm{~nm}) / \mathrm{CoFeB}(9 \mathrm{~nm}) /$ $\operatorname{Pt}(4 \mathrm{~nm})$, was also fabricated and characterized and is referred to as SL. The effects of pinholes on the interlayer coupling in our SFM samples is deemed to be negligible given that the first AF RKKY peak (which occurs at $t_{\mathrm{Ru}}=0.6 \mathrm{~nm}$ ) shows strong AF interlayer coupling as demonstrated in our earlier work [22].

The magnetic properties of the samples were examined using vibrating sample magnetometry (VSM) and a broadband vector network analyzer- ferromagnetic resonance spectrometer (VNA-FMR) for static and dynamic characterization respectively. The samples were prepared using a Southbay disk cutter to provide a 5-mm-diameter disk. VSM and VNAFMR measurements were undertaken with the magnetic field applied in the plane of the films at room temperature (see details in Appendix B 2 and B 3). The resonant adsorption was obtained from the VNA $S_{12}$ absorption parameter, which we then used to determine the resonant frequency of the samples. Figures 2(a)-2(d) shows examples of individual spectra measured using applied magnetic fields of 1, 4, and $7 \mathrm{kOe}$ for the SL, SFM1, SFM2, and SFM3 samples respectively. Figures 2(e)-2(h) combine a series of $S_{12}$ spectra to create $2 D$ maps of the resonance spectra as functions of applied field and frequency.

These data demonstrate that both the lower frequency AM and the higher frequency OM occur over a range of applied fields. The field dependence of the resonances is consistent across all samples. We observe in all cases that as the magnitude of applied field is decreased the OM reduces in amplitude compared to the AM. In SFMs, the OM is formed due to the influence of a FM $J_{\text {IEC }}$ and a difference in layer magnetization [2]. The presence of a FM layer with reduced magnetization is supported by the $M-H$ loop measurements presented in Figs. 2(e)-2(h) (bottom-left insets) where it is seen that the samples possess a lower saturation magnetization compared to the single $\mathrm{CoFeB}$ layer case of $\sim 1300 \mathrm{emu} / \mathrm{cm}^{3}$ for $\mathrm{CoFeB}$ thickness of both $4 \mathrm{~nm}$ (single layer) and $9 \mathrm{~nm}$ (SL: total magnetic layer). The saturation magnetization of each sample is presented in Table I. It is notable that an OM which is clearly separated from the AM is observed given the similarity in thickness of the two magnetic layers. Examining the spectra in detail shows that the frequency of the AM and OM differ for SFM1, SFM2 and SFM3. despite the subatomic change in $\mathrm{Ru}$ layer thickness. These data show the expected dependence of the RKKY interaction on the Ru NM spacer layer thickness and demonstrate the high sensitivity of the measurement. This 
(a)

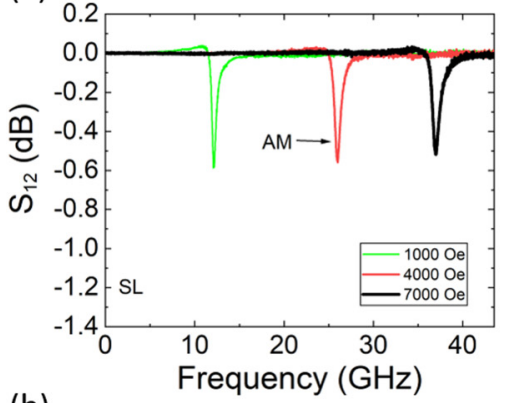

(b) 0.2

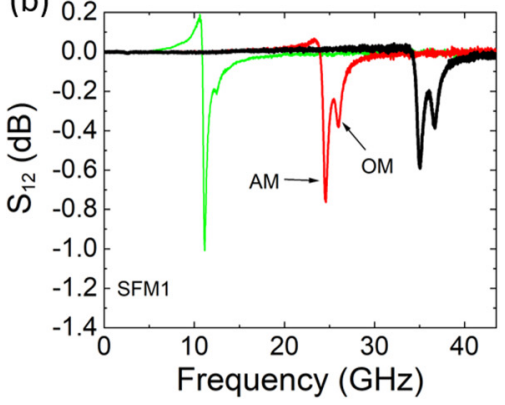

(c)
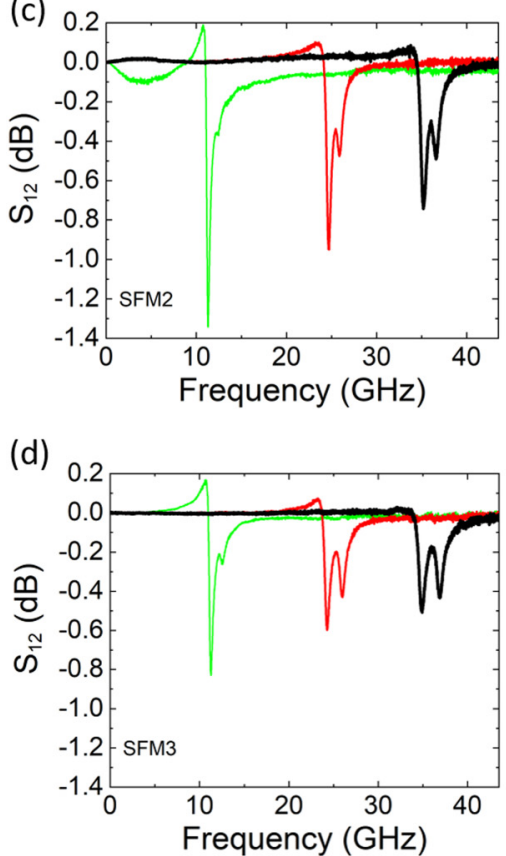

(e)

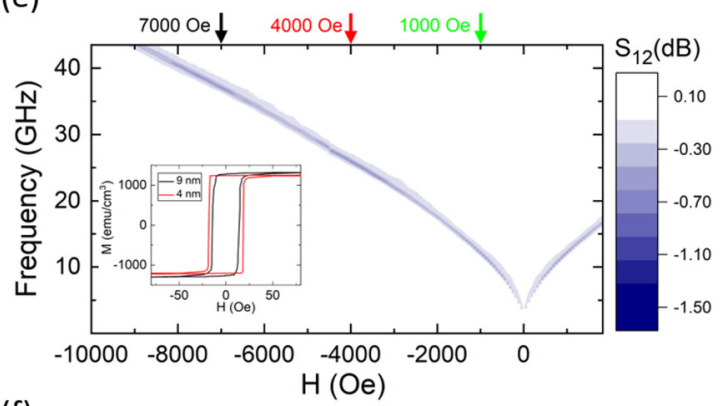

(f)

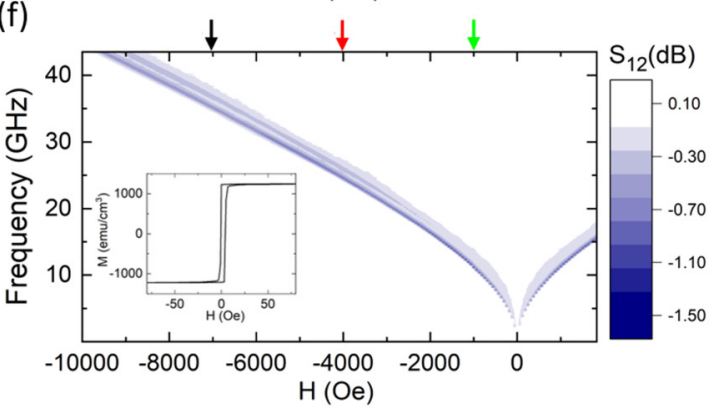

(g)

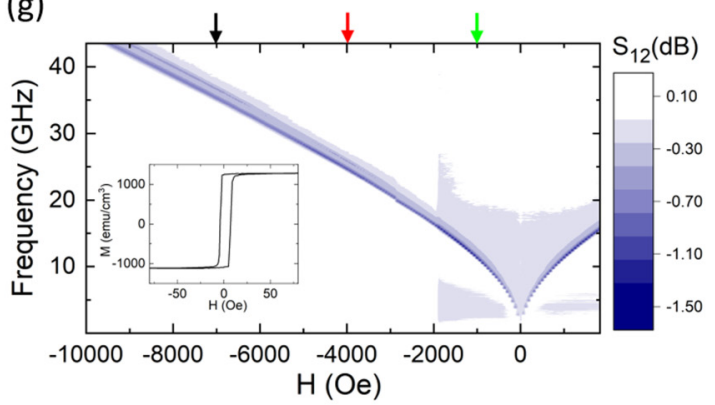

(h)

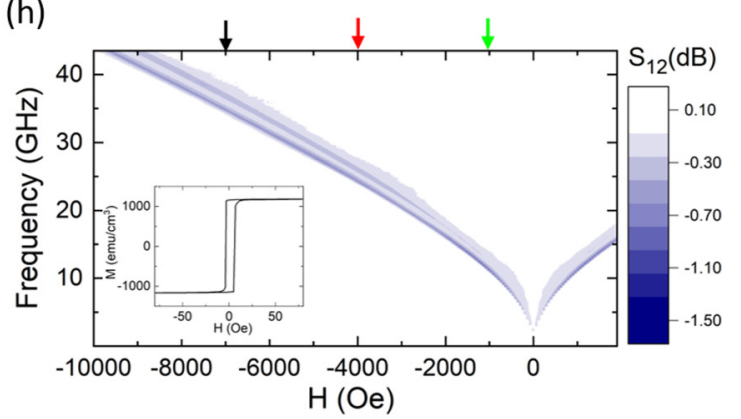

FIG. 2. Resonant dynamics of the single $\mathrm{CoFeB}$ layer (thickness $9 \mathrm{~nm}$ ) and synthetic ferromagnetic samples for a range of applied fields $(H)$. $\mathrm{S}_{12}$ absorption profiles for samples on the first FM maxima at varying applied field strength in the case (a) single layer, (b) SFM1, (c) SFM2, (d) SFM3. (e)-(h) Corresponding 2D resonance spectra. The insets show the in-plane $M-H$ hysteresis loops of each sample measured by VSM at room temperature. In (e), the $M-H$ loop of a single layer CoFeB film of thickness $4 \mathrm{~nm}$ is also presented (red).

extraordinary sensitivity to NM thickness is a key challenge in sample fabrication, with the data demonstrating that a high degree of control over the sample structure has been achieved.

\section{MICROMAGNETIC SIMULATION OF SFM RESONANT DYNAMICS}

Motivated by the hysteresis loop data where a reduction in saturation magnetization is observed relative to the single layer case (as shown in Table I) we use simulation to study the impacts of varying the difference in the layer magnetization and $J_{\text {IEC }}$ on the dynamics of the SFMs. We reproduced the $\mathrm{FM}_{1} / \mathrm{NM} / \mathrm{FM}_{2}$ structure of the sample with a micromagnetic model as shown in Fig. 3(a). Our simulations are implemented in the finite-difference GPU-accelerated program, MUMAX ${ }^{3}$ [38]. In order to minimize the demagnetization effects from the geometric edges, the dimension in the $x y$ plane is set to $128 \mathrm{~nm} \times 128 \mathrm{~nm}$ with periodic boundary conditions applied in the plane, and the overall system is discretized into $1 \mathrm{~nm} \times 1 \mathrm{~nm} \times 0.5 \mathrm{~nm}$ cuboid cells. The magnetic parameters were chosen to allow direct comparison between the numerical simulation and the experimental CoFeB SFM 


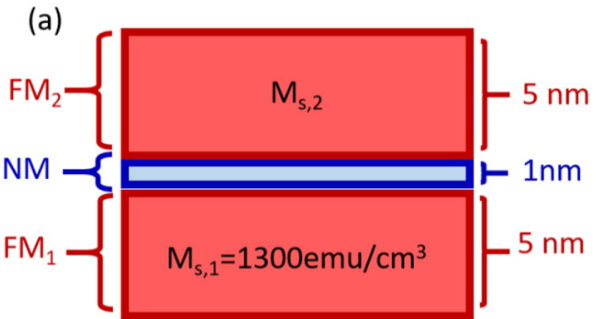

(c)

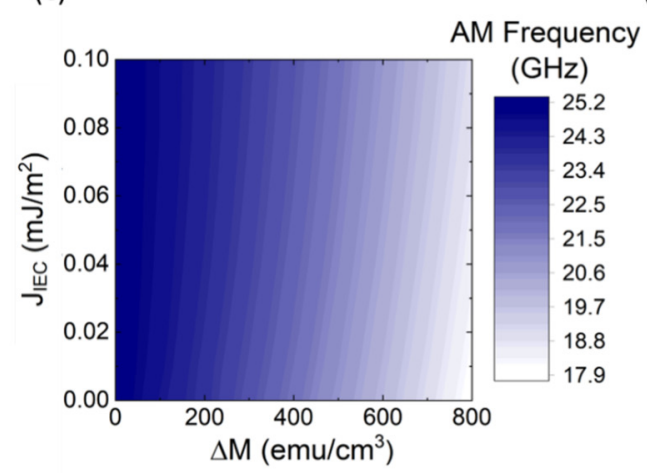

(e)

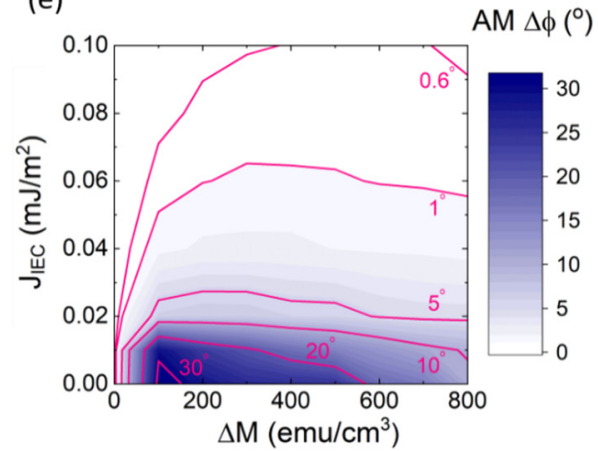

(b)

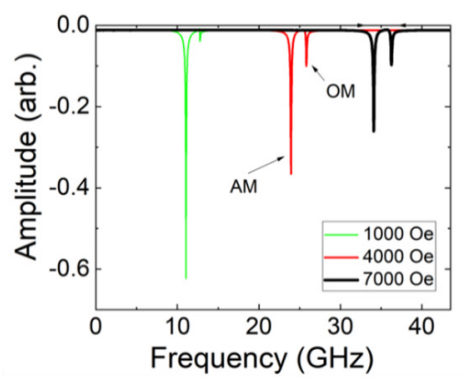

(d)

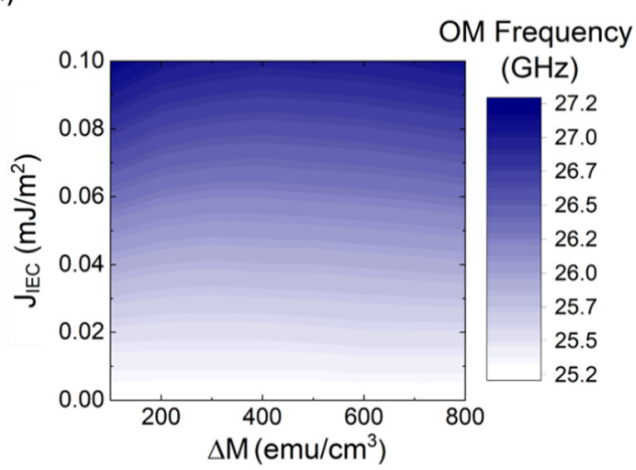

(f)

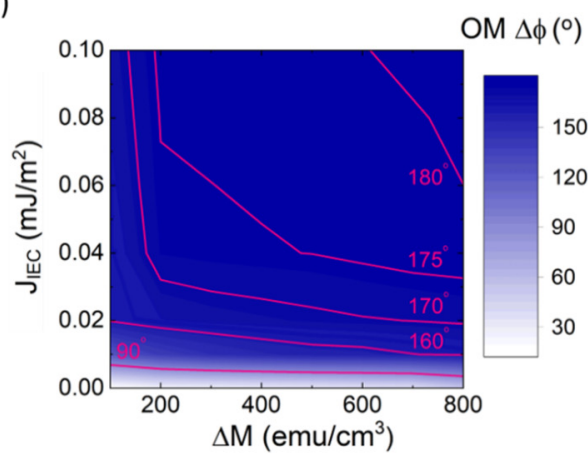

FIG. 3. Simulated dynamics of synthetic ferromagnets (SFMs). (a) Schematic of the simulated SFM structure, consisting of a $\mathrm{FM}_{1} / \mathrm{NM} / \mathrm{FM}_{2}$ trilayer. The layer magnetization of $\mathrm{FM}_{1}\left(M_{\mathrm{s}, 1}\right)$ is fixed while the layer magnetization of $\mathrm{FM}_{2}\left(M_{\mathrm{s}, 2}\right)$ is varied. (b) Simulated absorption spectrum of a SFM where $\Delta M=100 \mathrm{emu} / \mathrm{cm}^{3}$ and $J_{\text {IEC }}=0.1 \mathrm{~mJ} / \mathrm{m}^{2}$ at a range of different fields. (c)-(f) Impact of $\Delta M$ and $J_{\text {IEC }}$ at an externally applied field of $4 \mathrm{kOe}$ on the resonance frequency of the (c) AM, (d) OM, and on $\Delta \Phi$ of the resonances of the (e) AM and (f) OM.

samples. The exchange constant $\left(A_{\mathrm{ex}}\right)$ of the system was set to $A_{\text {ex }}=10^{-6} \mathrm{erg} / \mathrm{cm}$ and the saturation magnetization of $\mathrm{FM}_{1}$ was set to $M_{\mathrm{s}, 1}=1300 \mathrm{emu} / \mathrm{cm}^{3}$ [22]. The resonant frequencies were investigated for saturation magnetization of $\mathrm{FM}_{2}$ possessing a range of values between $M_{\mathrm{s}, 2}=500 \mathrm{emu} / \mathrm{cm}^{3}$ and $M_{\mathrm{s}, 2}=1200 \mathrm{emu} / \mathrm{cm}^{3}$, allowing OM dynamics as a function of $J_{\text {IEC }}$ to be explored. These parameters were chosen to

TABLE I. The saturation magnetization of the single layer $\mathrm{CoFeB}$ and the SFM samples. In the single layer case, the $M_{\mathrm{s}}$ of the 9- and 4-nm-thick samples were averaged.

\begin{tabular}{lc}
\hline \hline Sample & $M_{s}\left(\mathrm{emu} / \mathrm{cm}^{3}\right)$ \\
\hline Average single layer CoFeB & $1280 \pm 60$ \\
SFM1 & $1240 \pm 60$ \\
SFM2 & $1230 \pm 60$ \\
SFM3 & $1180 \pm 60$ \\
\hline \hline
\end{tabular}

be similar to literature reports for $\mathrm{CoFeB}$ trilayer structures $[22,26]$. We use the ringdown method [39] to extract the characteristic frequencies from the ferromagnetic resonance. The phase information of the system is determined by applying a time-dependent sinusoidal excitation $h_{\mathrm{exc}}(t)$ possessing frequency $f$ with $h_{\text {exc }}(t)=h_{0} \sin (2 \pi f t)$ with the time-resolved magnetization in each FM layer then subsequently recorded (see Appendix $\mathrm{C}$ for further details).

To treat this numerical simulation as representative of the samples, we must assume that $M_{\mathrm{s}, 1}$ is identical to that measured in single layer $\mathrm{CoFeB}$ while $M_{s, 2}$ is reduced. This is a reasonable hypothesis but deviations in the magnetic properties of $\mathrm{FM}_{1}$ from single layer $\mathrm{CoFeB}$ cannot be completely excluded due to mechanisms such as interdiffusion with the Ru layer $[40,41]$. Our XRR data demonstrate that no significant changes in layer density occurs. However, static measurements using vibrating sample magnetometry do show that the saturation magnetization of the SFM samples are reduced compared to the single layer $\mathrm{CoFeB}$ case, 
(a)

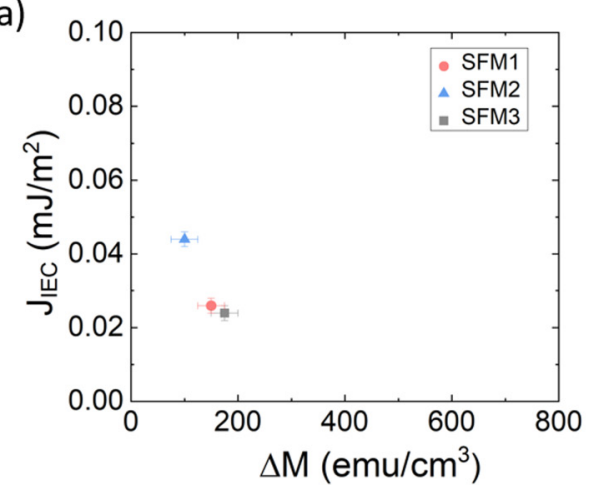

(b)

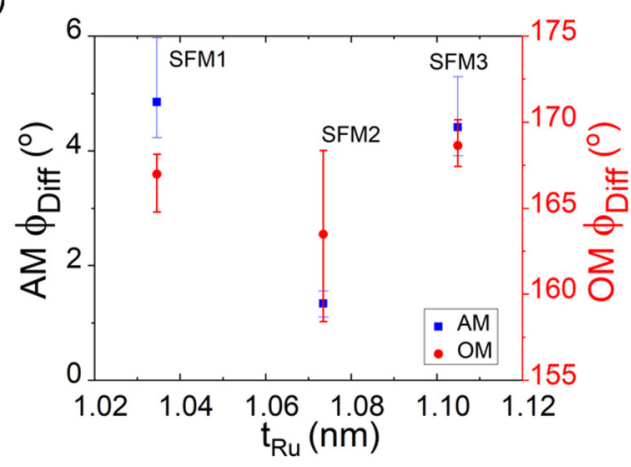

FIG. 4. Extracted parameters for the studied synthetic ferromagnets (SFMs) with differing $t_{\mathrm{Ru}}$. (a) Parameters matching the resonances of SFM samples plotted on the same scale as Figs. 3(c) and 3(d) to allow direct comparison with simulated cases. (b) $\Delta \Phi$ of the magnetic layers of the AM and OM for each SFM as extracted from the numerical simulation.

thus supporting the modeling of a structure with reduced magnetization [see bottom-left insets of Figs. 2(e)-2(h) and Table I].

Figure 3(b) shows that the key features of the resonance behavior exhibited by the samples can be accurately reproduced by the simulation with the formation of a double resonance observed along with a significant reduction of the optic mode intensity as the magnitude of the applied field is reduced. From Figs. 3(c)-3(f), a general assessment of the impact of the difference in layer magnetization and interlayer coupling strength on SFM dynamics can be made. Strikingly, it is shown that the resonant frequencies have orthogonal dependencies on $\Delta M$ and $J_{\text {IEC. }}$. Specifically the AM resonance is mostly dependent on $\Delta M$ while the OM resonance is governed mainly by $J_{\text {IEC }}$ strength. Indeed, the greatest reduction in AM resonance and enhancement in OM resonance is seen in cases where there is a greater $\Delta M$ and a higher $J_{\text {IEC }}$ respectively.

The simulations also allow the phase difference between the two resonating magnetic layers of each mode to be determined, as shown in Figs. 3(e) and 3(f). It is demonstrated that this phase behavior is impacted by both $\Delta M$ and $J_{\mathrm{IEC}}$, which contrasts with the dependence shown by the resonant frequencies. The simulation also reveals that the conventional in phase and out of phase understanding of the AM and OM does not fully capture the intricacy of the rich dynamics supported by a SFM system and that the resonances are better described through acousticlike/opticlike labels. Notably, in all simulated cases we do not achieve a truly out of phase $\left(180^{\circ}\right)$ optic mode. We see that the phase differences vary significantly from the AM/OM description in the case where the SFMs possess an extremely weak $J_{\mathrm{IEC}}\left(0-0.04 \mathrm{~mJ} / \mathrm{m}^{2}\right)$ and similar layer magnetizations. The simulations clearly demonstrate that the OM has a much greater sensitivity to these parameters than the AM. This is particularly seen in the weak coupling limit $\left(\sim 0.01 \mathrm{~mJ} / \mathrm{m}^{2}\right)$ as shown by Fig. 3(f) where the $\Delta \Phi$ transitions from a high phase difference $\left(\sim 180^{\circ}\right)$ quasioptic mode to a low phase difference $\left(<90^{\circ}\right)$ acousticlike mode.

\section{PARAMETER EXTRACTION}

The simulations allow us to understand the experimental data by comparing these numerical results with the measured resonance frequencies of the OM and AM peaks. Such an approach enables us to determine $J_{\mathrm{IEC}}$ and $\Delta M$ corresponding to our samples, as well as obtaining dynamic information on their phase behavior. The $J_{\mathrm{IEC}}$ and $\Delta M$ required to reproduce the resonances of each experimentally measured SFM are presented in Fig. 4(a) and Table II. We minimized the average residual between frequencies of the measured and simulated resonances for both modes to obtain the optimal parameters for the AM and OM for each sample. We demonstrate that the magnetization of the samples [bottom-left insets in Figs. 2(f) $-2(\mathrm{~h})$ and Table I] is consistent with the $\Delta M$ required to simulate the resonances of each sample, with SFM1 and SFM3 displaying a low saturation magnetization compared to SFM2. It is notable that although the magnetization differences are significantly lower than those demonstrated in the investigation performed by McKinnon et al. [26] we can measure and model these resonances. Furthermore, the magnitudes of the extracted $J_{\text {IEC }}\left(0.02-0.04 \mathrm{~mJ} / \mathrm{m}^{2}\right)$ are consistent with literature expectations [26] and also exhibit a behavior with Ru layer thickness that is consistent with that expected of the first RKKY FM coupling peak. That is, the highest $J_{\text {IEC }}$ is observed for SFM2 indicating that it is close to the maximum of the first RKKY FM peak with SFM1 and SFM3 to either side of the maximum.

The $\Delta M$ and $J_{\mathrm{IEC}}$ values extracted from fitting to the resonant frequencies can be used to obtain the phase behavior of the AM and OM mode for each SFM. The phase behavior of the AM and OM as a function of Ru thickness is shown in Fig. 4(b). In the case of SFM2 it is seen that despite possessing a higher $J_{\mathrm{IEC}}$, the OM $\Delta \Phi$ is lower than that seen in SFM1 and SFM3. This is a direct consequence of the lower $\Delta M$ in the case of SFM2. This analysis demonstrates how phase information from resonant modes can be obtained from VNAFMR laboratory based instrumentation and simulation. This

TABLE II. Parameters extracted from the numerical simulation for the studied SFM samples.

\begin{tabular}{lcc}
\hline \hline Sample & $\Delta M\left(\mathrm{emu} / \mathrm{cm}^{3}\right)$ & $J_{\text {IEC }}\left(\mathrm{mJ} / \mathrm{m}^{2}\right)$ \\
\hline SFM1 & $150 \pm 25$ & $0.026 \pm 0.002$ \\
SFM2 & $100 \pm 25$ & $0.044 \pm 0.002$ \\
SFM3 & $175 \pm 25$ & $0.024 \pm 0.002$ \\
\hline \hline
\end{tabular}


offers opportunities for future comparison with synchrotron techniques such as x-ray detected FMR [42] or reflectometry FMR [43].

\section{CONCLUSIONS}

In conclusion, we have demonstrated the experimental observation and numerical simulation of the resonant dynamics of synthetic ferromagnets (SFMs). We show that a well separated double resonance can be observed in samples with ferromagnetic layers of similar thickness, due to small differences in their magnetizations. The simulations have demonstrated a dependence of the acoustic and optic modes on (i) the difference in magnetization of the ferromagnetic layers and (ii) the interlayer exchange coupling. We show that an orthogonal dependence exists, where the AM exhibits a greater sensitivity to the magnetization difference while the $\mathrm{OM}$ is more dependent on the coupling strength. Thus, by comparing the measured resonance frequencies with simulations we describe a technique to determine the interlayer exchange coupling in these structures. Furthermore, using the parameters obtained we can extract, by numerical simulation, the phase behavior of the magnetic layers at each resonant mode, a property that is difficult to obtain experimentally other than via synchrotron techniques. We demonstrate that the conventional acoustical and optical description does not capture the intricacy of the dynamics exhibited by these structures. This work has direct application in the development of future exchange coupled spintronic materials with tailored properties for desired MRAM and high-frequency device applications.

The data that support the plots and other findings of this study are available from the corresponding authors upon reasonable request.

\section{ACKNOWLEDGMENTS}

The authors gratefully acknowledge the support of the UK EPRSC CDT Graphene NOWNANO through Grant No. EP/L01548X/1 and the involvement of the facilities of the Henry Royce Institute through EPSRC Grants No. EP/S019367/1 and No. EP/P025021/1. The authors would like to acknowledge the assistance given by Research IT and the use of the Computational Shared Facility at The University of Manchester. Y.L. acknowledges a scholarship supported by the China Scholarship Council.

\section{APPENDIX A: THIN-FILM FABRICATION METHOD}

Fabrication of the samples was undertaken using an AJA ATC 2200-V magnetron sputtering system. The substrates used were $\mathrm{Si} / \mathrm{SiO}_{2}$ where the oxide layer was $290 \mathrm{~nm}$. No deliberate substrate heating was used throughout deposition or as an annealing step. The overall structure of the metal layers was $\mathrm{Ta} / \mathrm{CoFeB} / \mathrm{Ru} / \mathrm{CoFeB}$ with a Pd cap employed. The deposition of the $\mathrm{Ta}, \mathrm{Co}_{0.2} \mathrm{Fe}_{0.6} \mathrm{~B}_{0.2}$, and Pd layers was carried out using DC magnetron sputtering from either elemental or, in the case of $\mathrm{CoFeB}$, alloy targets. The Ta was present to act as an adhesion layer. The $\mathrm{Ta}$ and $\mathrm{CoFeB}$ were deposited at a power of $20 \mathrm{~W}$, with the cap layer deposited at $100 \mathrm{~W}$.
The Ru layer sandwiched in the middle of the CoFeB layers was deposited through radio frequency (rf) sputtering at a power of $75 \mathrm{~W}$. The deposition time of the Ru layers were 90, 100, and 110 s for SFM1, SFM2, and SFM3 respectively. This allowed the Ru layer thicknesses to be determined from a calibration of thickness vs deposition time. The base pressure prior to deposition was $10^{-8}$ Torr and no in situ magnetic fields applied. The working pressure of the $\mathrm{Ar}^{+}$gas was between 3 and 4 mTorr.

\section{APPENDIX B: CHARACTERIZATION METHODS}

\section{Structure characterization}

The structural properties of the layers were obtained using X-ray reflectivity (XRR). The measurements employed a Rigaku Smart Lab system using a 3-KW copper K alpha source. All measurements were performed over a $2 \theta$ range $0.1-8.0^{\circ}$ with a step size of 0.01 . The data were fitted to a Fresnel model by means of the Parratt recursive algorithm [36] using the GENX simulation package [37]. The fitting was undertaken until the reduced $\chi^{2}$ figure of merit was optimized. These measurements provided information on the thickness, roughness, and density of the constituent layers. Figure 5 presents the fitting to the x-ray measurements and the corresponding scattering length density (SLD) for each sample.

\section{Magnetic measurements}

Magnetization vs applied magnetic field $(M-H)$ loops were measured by vibrating sample magnetometry (VSM) using a Microsense Model 10 vector VSM. The samples were prepared using a Southbay disk cutter to provide an 8-mm disk for the VSM measurement. These measurements were done using an in-plane applied magnetic field up to $20 \mathrm{kOe}$ to ensure saturation of the magnetic domain structure. Background correction was performed by performing a linear fit to the saturated region of the hysteresis loop, with its gradient determining the diamagnetic response present in the measurement.

\section{Ferromagnetic resonance measurements}

The high frequency properties of the films were characterized using a vector network analyzer ferromagnetic resonance (VNA-FMR) setup. This setup employed a picoprobe waveguide and a Keysight VNA N5224A which supports frequency measurements in the range $10 \mathrm{MHz}-43.5 \mathrm{GHz}$. The probes are contacted to a ground-signal-ground waveguide placed within a magnetic field of up to $1.4 \mathrm{~T}$ provided by a GMW electromagnet. The sample was placed face-down upon the waveguide in a flip-chip manner for measurement, where a natural air barrier was sufficient to prevent the shorting of the waveguide. The operating principle of this method is that the magnetic configuration of a film under test will be perturbed by an rf magnetic field due to the electric signals traversing the waveguide. When the frequency of the perturbing magnetic field is equal to the resonant frequency of the thin film, a significant absorption of this rf energy takes place which causes the transmission of energy through the waveguide (measured through the $S_{12}$ parameter) to decrease [3,44]. This method 
(a)

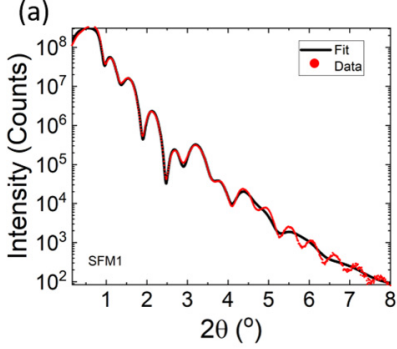

(b)

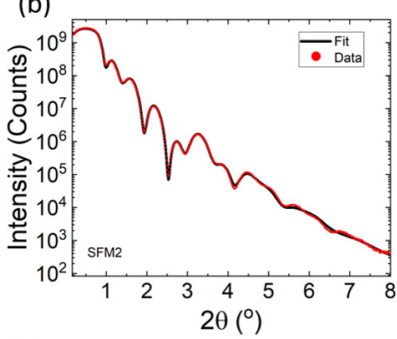

(c)

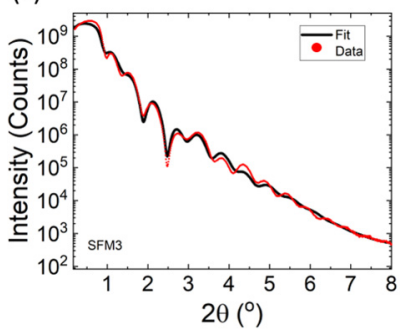

(d)

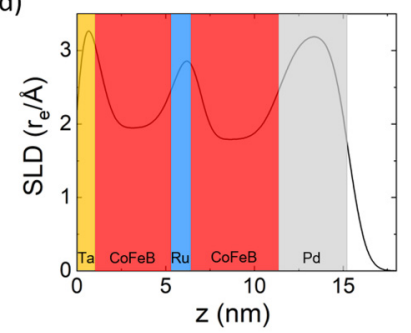

(e)

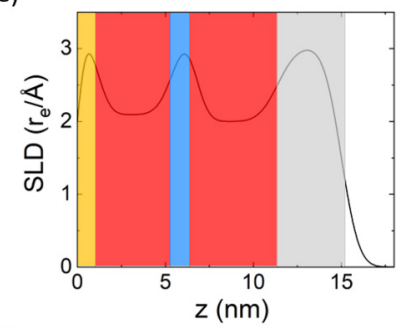

(f)

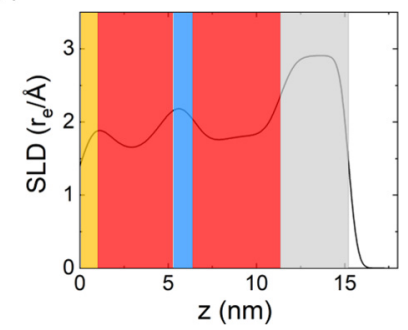

FIG. 5. Structural characterization of synthetic ferromagnets performed using XRR. The XRR data set and fit for Ru layer thickness (a) SFM1 $\left(t_{\mathrm{Ru}}=1.03 \mathrm{~nm}\right)$, (b) SFM1 $\left(t_{\mathrm{Ru}}=1.07 \mathrm{~nm}\right)$, and (c) SFM3 $\left(t_{\mathrm{Ru}}=1.10 \mathrm{~nm}\right)$. Corresponding SLD plots as derived from XRR measurement as a function of distance from substrate $(z)$ for (a) SFM1, (b) SFM2, (c) SFM3. The overlaying colors represent the different layers of the stacks namely Ta (orange), $\mathrm{CoFeB}$ (red), $\mathrm{Ru}$ (blue), and Pd (grey).

was carried out by performing frequency sweeps for a range of applied external fields as opposed to the conventional field sweeps for set frequencies.

\section{APPENDIX C: SIMULATION METHODS}

Based on the micromagnetic model, resonate dynamics of magnetization is characterized by solving the Landau-Lifshitz equation [45]

$$
\frac{\partial \mathbf{m}}{\partial t}=\frac{\gamma}{1+\alpha^{2}}\left\{\mathbf{m} \times \mathbf{H}_{\mathrm{eff}}+\alpha\left[\mathbf{m} \times\left(\mathbf{m} \times \mathbf{H}_{\mathrm{eff}}\right)\right]\right\},
$$

where $\mathbf{m}$ indicates the normalized magnetization in constant magnitude $M_{\mathrm{S}}=\mathbf{M} / \mathbf{m}$, with the damping parameter $\alpha$ and the gyromagnetic ratio of the electron $\gamma$. The effective field $\mathbf{H}_{\text {eff }}$ applied on the magnetization is defined as $\mathbf{H}_{\mathrm{eff}}=-\mu_{0}^{-1} \delta E / \delta \mathbf{M}$, which is based on the total energy $E$ that comprises the symmetric exchange interaction, the Zeeman coupling, the magnetostatic interaction, and the Ruderman-Kittel-Kasuya-Yoshida (RKKY) interlayer exchange coupling. In order to guarantee the accuracy of the dynamic simulations, the Landau-Lifshitz equation is solved by Runge-Kutta method (RK45) with a maximum time step $10^{-6} \mathrm{~ns}$. In the dynamic simulations we use the damping parameter $\alpha=0.01$. Note that much smaller time steps and different damping parameters in the underdamped range $(0<$ $\alpha<1$ ) have been tested, and the results showed no difference in quantity.

Our simulations are implemented by the finite-difference GPU-accelerated micromagnetics program MUMAX ${ }^{3}$ [38]. Unless specified otherwise, the synthetic ferromagnet (SFM) is described by a trilayer structure with $\mathrm{FM}_{1}(5 \mathrm{~nm}) / \mathrm{NM}$ $(1 \mathrm{~nm}) / \mathrm{FM}_{2}(5 \mathrm{~nm})$. In order to minimize the demagnetization effects from the geometric edges, the dimension in the $x y$ plane is set to $128 \mathrm{~nm} \times 128 \mathrm{~nm}$ with periodic boundary conditions applied in the plane, and the overall system is discretized into $1 \mathrm{~nm} \times 1 \mathrm{~nm} \times 0.5 \mathrm{~nm}$ cuboid cells. In the ringdown method [39] the system is initialized to a field-polarized state, followed by a weak magnetic excitation $h_{\text {exc }}$ applied for $10 \mathrm{~ns}$ to perturb it from its equilibrium, and the magnetization dynamics is sampled and recorded with a time step of $10^{-4} \mathrm{~ns}$, where at least 100 steps are solved between the recorded data points. Subsequently, a fast Fourier transform (FFT) is used to obtain the amplitude spectrum of the averaged magnetization, and the characterized frequency is extracted from the measured time domain.
[1] I. S. Maksymov and M. Kostylev, Broadband stripline ferromagnetic resonance spectroscopy of ferromagnetic films, multilayers and nanostructures, Physica E (Amsterdam, Neth.) 69, 253 (2015).

[2] B. Heinrich and J. F. Cochran, Ultrathin metallic magnetic films: magnetic anisotropies and exchange interactions, Adv. Phys. 42, 523 (1993).

[3] M. Farle, Ferromagnetic resonance of ultrathin metallic layers, Rep. Prog. Phys. 61, 755 (1998).

[4] S. Li, Q. Li, J. Xu, S. Yan, G.-X. Miao, S. Kang, Y. Dai, J. Jiao, and Y. Lü, Tunable optical mode ferromagnetic resonance in $\mathrm{FeCoB} / \mathrm{Ru} / \mathrm{FeCoB}$ synthetic antiferromagnetic trilayers un- der uniaxial magnetic anisotropy, Adv. Funct. Mater. 26, 3738 (2016).

[5] J. Walowski and M. Münzenberg, Perspective: Ultrafast magnetism and THz spintronics, J. Appl. Phys. 120, 140901 (2016).

[6] G. X. Miao, M. Münzenberg, and J. S. Moodera, Tunneling path toward spintronics, Rep. Prog. Phys. 74, 036501 (2011).

[7] Y. Wu, M. Elyasi, X. Qiu, M. Chen, Y. Liu, L. Ke, and $\mathrm{H}$. Yang, High-performance $\mathrm{THz}$ emitters based on ferromagnetic/nonmagnetic heterostructures, Adv. Mater. 29, 1603031 (2017).

[8] T. Seifert, S. Jaiswal, U. Martens, J. Hannegan, L. Braun, P. Maldonado, F. Freimuth, A. Kronenberg, J. Henrizi, I. Radu, 
E. Beaurepaire, Y. Mokrousov, P. M. Oppeneer, M. Jourdan, G. Jakob, D. Turchinovich, L. M. Hayden, M. Wolf, M. Münzenberg, M. Kläui, and T. Kampfrath, Efficient metallic spintronic emitters of ultrabroadband terahertz radiation, Nat. Photonics 10, 483 (2016).

[9] T. Jungwirth, X. Marti, P. Wadley, and J. Wunderlich, Antiferromagnetic spintronics, Nat. Nanotechnol. 11, 231 (2016).

[10] M. B. Jungfleisch, W. Zhang, and A. Hoffmann, Perspectives of antiferromagnetic spintronics, Phys. Lett. A 382, 865 (2018).

[11] A. Hoffmann, Spin pumping gathers speed, Science 368, 135 (2020).

[12] R. A. Duine, K. J. Lee, S. S. P. Parkin, and M. D. Stiles, Synthetic antiferromagnetic spintronics, Nat. Phys. 14, 217 (2018).

[13] S. S. P. Parkin and D. Mauri, Spin engineering: Direct determination of the ruderman-kittel-kasuya-yosida far-field range function in ruthenium, Phys. Rev. B 44, 7131 (1991).

[14] P. Grünberg, R. Schreiber, Y. Pang, U. Walz, M. B. Brodsky, and H. Sowers, Layered magnetic structures: Evidence for antiferromagnetic coupling of Fe layers across $\mathrm{Cr}$ interlayers, J. Appl. Phys. 61, 3750 (1987).

[15] M. N. Baibich, J. M. Broto, A. Fert, F. N. Van Dau, F. Petroff, P. Eitenne, G. Creuzet, A. Friederich, and J. Chazelas, Giant Magnetoresistance of (001)Fe/(001)Cr Magnetic Superlattices, Phys. Rev. Lett. 61, 2472 (1988).

[16] A. Fert, Origin, development, and future of spintronics (nobel lecture), Angew. Chem. Int. Ed. 47, 5956 (2008).

[17] S. A. Wolf, D. D. Awschalom, R. A. Buhrman, J. M. Daughton, S. Von Molnár, M. L. Roukes, A. Y. Chtchelkanova, and D. M. Treger, Spintronics: A spin-based electronics vision for the future, Science 294, 1488 (2001).

[18] P. Bruno, Theory of interlayer magnetic coupling, Phys. Rev. B 52, 411 (1995).

[19] P. Bruno and C. Chappert, Oscillatory Coupling between Ferromagnetic Layers Separated by a Nonmagnetic Metal Spacer, Phys. Rev. Lett. 67, 1602 (1991).

[20] Q. Leng, V. Cros, R. Schäfer, A. Fuss, P. Grünberg, and W. Zinn, Interlayer coupling across noble metal spacers, J. Magn. Magn. Mater. 126, 367 (1993).

[21] S. S. P. Parkin, R. Bhadra, and K. P. Roche, Oscillatory Magnetic Exchange Coupling through Thin Copper Layers, Phys. Rev. Lett. 66, 2152 (1991).

[22] H. J. Waring, N. A. B. Johansson, I. J. Vera-Marun, and T. Thomson, Zero-field optic mode beyond $20 \mathrm{GHz}$ in a synthetic antiferromagnet, Phys. Rev. Appl. 13, 034035 (2020).

[23] P. Bruno, Theory of interlayer exchange interactions in magnetic multilayers, J. Phys.: Condens. Matter 11, 9403 (1999).

[24] S. Sorokin, R. A. Gallardo, C. Fowley, K. Lenz, A. Titova, G. Y. P. Atcheson, G. Dennehy, K. Rode, J. Fassbender, J. Lindner, and A. M. Deac, Magnetization dynamics in synthetic antiferromagnets: Role of dynamical energy and mutual spin pumping, Phys. Rev. B 101, 144410 (2020).

[25] T. Devolder, E. Liu, J. Swerts, S. Couet, T. Lin, S. Mertens, A. Furnemont, G. Kar, and J. De Boeck, Ferromagnetic resonance study of composite $\mathrm{Co} / \mathrm{Ni}$ - FeCoB free layers with perpendicular anisotropy, Appl. Phys. Lett. 109, 142408 (2016).

[26] T. McKinnon, P. Omelchenko, B. Heinrich, and E. Girt, FMR study of interlayer exchange coupling in $\mathrm{FeCoB}|\mathrm{Ta}| \mathrm{FeCoB}$ trilayers with in-plane anisotropy, J. Appl. Phys. 123, 223903 (2018).

[27] T. McKinnon and E. Girt, Exchange coupling in $\mathrm{FeCoB} / \mathrm{Ru}$, $\mathrm{Mo} / \mathrm{FeCoB}$ trilayer structures, Appl. Phys. Lett. 113, 192407 (2018).

[28] S. Yakata, H. Kubota, T. Seki, K. Yakushiji, A. Fukushima, S. Yuasa, and K. Ando, Enhancement of thermal stability using ferromagnetically coupled synthetic free layers in $\mathrm{MgO}$ based magnetic tunnel junctions, IEEE Trans. Magn. 46, 2232 (2010).

[29] I. Yulaev, M. V. Lubarda, S. Mangin, V. Lomakin, and E. E. Fullerton, Spin-transfer-torque reversal in perpendicular anisotropy spin valves with composite free layers, Appl. Phys. Lett. 99, 132502 (2011).

[30] T. J. Klemmer, K. A. Ellis, L. H. Chen, B. Van Dover, and S. Jin, Ultrahigh frequency permeability of sputtered Fe-Co-B thin films, J. Appl. Phys. 87, 830 (2000).

[31] T. Burkert, L. Nordström, O. Eriksson, and O. Heinonen, Giant Magnetic Anisotropy in Tetragonal FeCo Alloys, Phys. Rev. Lett. 93, 027203 (2004).

[32] S. Li, G. X. Miao, D. Cao, Q. Li, J. Xu, Z. Wen, Y. Dai, S. Yan, and Y. Lü, Stress-enhanced interlayer exchange coupling and optical-mode FMR frequency in self-bias Fe$\mathrm{CoB} / \mathrm{Ru} / \mathrm{FeCoB}$ trilayers, ACS Appl. Mater. Interfaces 10, 8853 (2018).

[33] X. Liu, W. Zhang, M. J. Carter, and G. Xiao, Ferromagnetic resonance and damping properties of $\mathrm{CoFeB}$ thin films as free layers in MgO-based magnetic tunnel junctions, J. Appl. Phys. 110, 033910 (2011).

[34] A. Conca, E. T. Papaioannou, S. Klingler, J. Greser, T. Sebastian, B. Leven, J. Lösch, and B. Hillebrands, Annealing influence on the gilbert damping parameter and the exchange constant of $\mathrm{CoFeB}$ thin films, Appl. Phys. Lett. 104, 182407 (2014).

[35] C. Bilzer, T. Devolder, J.-V. Kim, G. Counil, C. Chappert, S. Cardoso, and P. P. Freitas, Study of the dynamic magnetic properties of soft CoFeB films, J. Appl. Phys. 100, 053903 (2006).

[36] L. G. Parratt, Surface studies of solids, Phys. Rev. 95, 359 (1954).

[37] M. Björck and G. Andersson, GenX: An extensible x-ray reflectivity refinement program utilizing differential evolution, J. Appl. Crystallogr. 40, 1174 (2007).

[38] A. Vansteenkiste, J. Leliaert, M. Dvornik, M. Helsen, F. GarciaSanchez, and B. Van Waeyenberge, The design and verification of MuMax3, AIP Adv. 4, 107133 (2014).

[39] A. Baker, M. Beg, G. Ashton, M. Albert, D. Chernyshenko, W. Wang, S. Zhang, M. A. Bisotti, M. Franchin, C. L. Hu, R. Stamps, T. Hesjedal, and H. Fangohr, Proposal of a micromagnetic standard problem for ferromagnetic resonance simulations, J. Magn. Magn. Mater. 421, 428 (2017).

[40] Y. H. Wang, W. C. Chen, S. Y. Yang, K. H. Shen, C. Park, M. J. Kao, and M. J. Tsai, Interfacial and annealing effects on magnetic properties of CoFeB thin films, J. Appl. Phys. 99, 08M307 (2006).

[41] S. Y. Jang, S. H. Lim, and S. R. Lee, Magnetic dead layer in amorphous $\mathrm{CoFeB}$ layers with various top and bottom structures, J. Appl. Phys. 107, 09C707 (2010).

[42] G. B. G. Stenning, L. R. Shelford, S. A. Cavill, F. Hoffmann, M. Haertinger, T. Hesjedal, G. Woltersdorf, G. J. Bowden, S. A. 
Gregory, C. H. Back, P. A. J. De Groot, and G. V. Van Der Laan, Magnetization dynamics in an exchange-coupled $\mathrm{NiFe} / \mathrm{CoFe}$ bilayer studied by $\mathrm{x}$-ray detected ferromagnetic resonance, New J. Phys. 17, 013019 (2015).

[43] D. M. Burn, S. L. Zhang, G. Q. Yu, Y. Guang, H. J. Chen, X. P. Qiu, G. Van Der Laan, and T. Hesjedal, Depth-Resolved Magnetization Dynamics Revealed by X-Ray Reflectome- try Ferromagnetic Resonance, Phys. Rev. Lett. 125, 137201 (2020).

[44] C. Kittel, On the theory of ferromagnetic resonance absorption, Phys. Rev. 73, 155 (1948).

[45] L. D. Landau and E. Lifshitz, Theory of the dispersion of magnetic permeability in ferromagnetic bodies, Phys. Z. Sowietunion 8, 153 (1935). 